\title{
An Outer Bound for the Gaussian MIMO Broadcast Channel With Common and Private Messages
}

\author{
Ersen Ekrem, Student Member, IEEE, and Sennur Ulukus, Member, IEEE
}

\begin{abstract}
We consider the Gaussian multiple-input multiple-output (MIMO) broadcast channel with common and private messages. We obtain an outer bound for the capacity region of this channel. To this end, we show that a parallel Gaussian broadcast channel can be constructed from any given Gaussian MIMO broadcast channel by using the generalized singular value decomposition and a relaxation on the power constraint for the channel input. Due to this relaxation of the power constraint, the capacity region of the constructed parallel channel, which is known, provides an outer bound for the capacity region of the original channel. We show that this outer bound is within a finite gap of the capacity region by comparing it with an achievable rate region that can be obtained either by using dirty-paper coding or by using a variation of the zero-forcing scheme.
\end{abstract}

Index Terms - Common messages, Gaussian MIMO broadcast channels, generalized singular value decomposition, outer bound.

\section{INTRODUCTION}

$\mathbf{W}$ E study the two-user Gaussian multiple-input multiple-output (MIMO) broadcast channel for the scenario where the transmitter sends a private message to each user in addition to a common message which is directed to both users. The capacity region for this scenario, i.e., the capacity region of the Gaussian MIMO broadcast channel with common and private messages, is unknown. However, when one of these three messages is absent, the corresponding capacity region is known. In particular, the capacity region is known when there is no common message, i.e., each user gets only a private message [2], and for the degraded message set case, i.e., there is a common message directed to both users, and only one of the users gets a private message [3], [4].

The first work that considers the Gaussian MIMO broadcast channel with common and private messages is [5], which proposed an achievable scheme by using a standard Gaussian coding scheme for the common message, and dirty-paper coding (DPC) for the private messages. The corresponding achievable rate region is called the DPC region. In addition, Jindal and Goldsmith [5] obtain the capacity region when the Gaussian

Manuscript received May 04, 2011; revised April 28, 2012; accepted June 23, 2012. Date of publication July 11, 2012; date of current version October 16,2012 . This work was supported by the National Science Foundation under Grants CCF 07-29127, CNS 09-64632, CCF 09-64645, and CCF 10-18185. This paper was presented in part at the 2010 IEEE Global Communications Conference.

The authors are with the Department of Electrical and Computer Engineering, University of Maryland, College Park, MD 20742 USA (e-mail: ersen@umd edu; ulukus@umd.edu).

Communicated by S. Jafar, Associate Editor for Communications.

Digital Object Identifier 10.1109/TIT.2012.2208175
MIMO broadcast channel is equivalent to a set of parallel independent Gaussian channels by using results from [6].

The Gaussian MIMO broadcast channel with common and private messages is further studied in [3] and [4], where the partial optimality of the DPC region [5] is shown. Weingarten et al. [3], [4] first propose an outer bound for the capacity region, and then show that it is tight on certain subregions of the capacity region by showing that it matches the DPC region given in [5]. Moreover, Weingarten et al. [3], [4] show that for a given common message rate, the private message sum capacity is attained by the DPC region in [5]. Finally, they [3], [4] show the optimality of the DPC region in [5] when the common message rate is beyond a certain threshold. However, this result holds when the channel input is subject to a covariance constraint, and cannot be extended to the case where the channel input is subject to a total power constraint [7]. Recently, another outer bound is given in [7] and [8], where we show that extending the DPC region in the common message rate direction by a fixed amount is an outer bound for the capacity region. However, this fixed amount, i.e., the gap, is not necessarily finite, since it scales with the available power.

In this study, we obtain a new outer bound for the capacity region of the Gaussian MIMO broadcast channel with common and private messages, where this outer bound, irrespective of the available power, is within a finite gap of the capacity region. ${ }^{1}$ We first show that we can construct a parallel Gaussian broadcast channel from any given Gaussian MIMO broadcast channel such that the capacity region of this constructed parallel Gaussian broadcast channel includes the capacity region of the original Gaussian MIMO broadcast channel. To construct such a parallel channel, we use the generalized singular value decomposition (GSVD) [11] on the channel gain matrices of the Gaussian MIMO broadcast channel and also relax the power constraint on the channel input. This relaxation on the power constraint enlarges the capacity region during the transformation of the Gaussian MIMO broadcast channel into a parallel Gaussian broadcast channel. Consequently, the capacity region of the constructed parallel channel, which is known due to [6], provides us an outer bound for the capacity region of the

\footnotetext{
${ }^{1}$ In the literature, a finite gap result usually refers to, e.g., [9], [10], the existence of inner and outer bounds on the capacity region such that there is a finite gap between them, and the corresponding gap is universal in the sense that it does not depend on the available power and channel gains. On the other hand, here, we use finite gap result in a slightly different context. Here, similar to [9] and [10], a finite gap result implies the existence of the inner and outer bounds within a finite gap which does not depend on the available power. However, as opposed to [9] and [10], here this finite gap depends on the channel gains. It is well-known [10, Section II-E] that for MIMO Gaussian models, obtaining finite gap results with gap being independent of both the available power and the channel gains is difficult.
} 
Gaussian MIMO channel. Next, we show that this outer bound is within a finite gap of the capacity region of the Gaussian MIMO broadcast channel by comparing it with an inner bound that can be attained either by using the DPC region or by using a variation of the zero-forcing (ZF) scheme. Finally, by means of this finite gap result, we obtain the degrees of freedom (DoF) region of the Gaussian MIMO broadcast channel with common and private messages.

\section{Channel Model And Definitions}

The Gaussian MIMO broadcast channel is defined by

$$
\begin{aligned}
\mathbf{Y}_{1} & =\mathbf{H}_{1} \mathbf{X}+\mathbf{N}_{1} \\
\mathbf{Y}_{2} & =\mathbf{H}_{2} \mathbf{X}+\mathbf{N}_{2}
\end{aligned}
$$

where the channel input $\mathbf{X}$ is a $t \times 1$ column vector, $\mathbf{H}_{j}$ is the $j$ th user's channel gain matrix of size $r_{j} \times t, \mathbf{Y}_{j}$ is the channel output of the $j$ th user which is an $r_{j} \times 1$ column vector, and the Gaussian random vector $\mathbf{N}_{j}$ is of size $r_{j} \times 1$ with an identity covariance matrix. The channel input is subject to an average power constraint as follows:

$$
E\left[\mathbf{X}^{\top} \mathbf{X}\right]=\operatorname{tr}\left(E\left[\mathbf{X} \mathbf{X}^{\top}\right]\right) \leq P .
$$

We study the Gaussian MIMO broadcast channel for the scenario where the transmitter sends a common message to both users, and a private message to each user. We call the channel model arising from this scenario the Gaussian MIMO broadcast channel with common and private messages. An $\left(n, 2^{n R_{0}}, 2^{n R_{1}}, 2^{n R_{2}}\right)$ code for this channel consists of three message sets $\mathcal{W}_{0}=$ $\left\{1, \ldots, 2^{n R_{0}}\right\}, \mathcal{W}_{1}=\left\{1, \ldots, 2^{n R_{1}}\right\}, \mathcal{W}_{2}=\left\{1, \ldots, 2^{n R_{2}}\right\}$, one encoder $f_{n}: \mathcal{W}_{0} \times \mathcal{W}_{1} \times \mathcal{W}_{2} \rightarrow \mathcal{X}^{n}$, one decoder at each receiver $g_{n}^{j}: \mathcal{Y}_{j}^{n} \rightarrow \mathcal{W}_{0} \times \mathcal{W}_{j}, j=1,2$. The probability of error is defined as $P_{e}^{n}=\max \left\{P_{e 1}^{n}, P_{e 2}^{n}\right\}$, where $P_{e j}=\operatorname{Pr}\left[g_{n}^{j}\left(f_{n}\left(W_{0}, W_{1}, W_{2}\right)\right) \neq\left(W_{0}, W_{j}\right)\right], \quad j=1,2$, and $W_{j}$ denotes the message which is a uniformly distributed random variable in $\mathcal{W}_{j}, j=0,1,2$. A rate triple $\left(R_{0}, R_{1}, R_{2}\right)$ is said to be achievable if there is an $\left(n, 2^{n R_{0}}, 2^{n R_{1}}, 2^{n R_{2}}\right)$ code with $\lim _{n \rightarrow \infty} P_{e}^{n}=0$. The capacity region $\mathcal{C}(P)$ is defined as the convex closure of all achievable rate triples $\left(R_{0}, R_{1}, R_{2}\right)$.

Now, we present the achievable rate region, hereafter called the DPC region, given in [5]. In this achievable scheme, the common message is encoded by a standard Gaussian codebook, and the private messages are encoded by DPC. Since a DPC scheme is used to encode the private messages, one of the users observes an interference-free link depending on the encoding order at the transmitter. We next define

$$
\begin{aligned}
R_{0 j}\left(\mathbf{K}_{0}, \mathbf{K}_{1}, \mathbf{K}_{2}\right) & =\frac{1}{2} \log \frac{\left|\mathbf{H}_{j}\left(\mathbf{K}_{0}+\mathbf{K}_{1}+\mathbf{K}_{2}\right) \mathbf{H}_{j}^{\top}+\mathbf{I}\right|}{\left|\mathbf{H}_{j}\left(\mathbf{K}_{1}+\mathbf{K}_{2}\right) \mathbf{H}_{j}^{\top}+\mathbf{I}\right|} \\
R_{1}\left(\mathbf{K}_{1}, \mathbf{K}_{2}\right) & =\frac{1}{2} \log \frac{\left|\mathbf{H}_{1}\left(\mathbf{K}_{1}+\mathbf{K}_{2}\right) \mathbf{H}_{1}^{\top}+\mathbf{I}\right|}{\left|\mathbf{H}_{1} \mathbf{K}_{2} \mathbf{H}_{1}^{\top}+\mathbf{I}\right|} \\
R_{2}\left(\mathbf{K}_{2}\right) & =\frac{1}{2} \log \left|\mathbf{H}_{2} \mathbf{K}_{2} \mathbf{H}_{2}+\mathbf{I}\right|
\end{aligned}
$$

where $\mathbf{K}_{0}, \mathbf{K}_{1}$, and $\mathbf{K}_{2}$ denote the covariance matrices allotted for the common message, the first user's private message, and the second user's private message, respectively. The DPC region is stated in the following theorem.

Theorem 1 ([5]): The rate triples $\left(R_{0}, R_{1}, R_{2}\right)$ lying in the region ${ }^{2}$

$$
\mathcal{R}^{\mathrm{DPC}}(P)=\operatorname{conv}\left(\mathcal{R}_{1}^{\mathrm{DPC}}(P) \cup \mathcal{R}_{2}^{\mathrm{DPC}}(P)\right)
$$

are achievable, where conv is the convex hull operator, $\mathcal{R}_{1}^{\mathrm{DPC}}(P)$ consists of rate triples $\left(R_{0}, R_{1}, R_{2}\right)$ satisfying

$$
\begin{aligned}
R_{0}+R_{1} \leq & R_{01}\left(\mathbf{K}_{0}, \mathbf{K}_{1}, \mathbf{K}_{2}\right)+R_{1}\left(\mathbf{K}_{1}, \mathbf{K}_{2}\right) \\
R_{0}+R_{2} \leq & R_{02}\left(\mathbf{K}_{0}, \mathbf{K}_{1}, \mathbf{K}_{2}\right)+R_{2}\left(\mathbf{K}_{2}\right) \\
R_{0}+R_{1}+R_{2} \leq & \min _{j=1,2} R_{0 j}\left(\mathbf{K}_{0}, \mathbf{K}_{1}, \mathbf{K}_{2}\right)+R_{1}\left(\mathbf{K}_{1}, \mathbf{K}_{2}\right) \\
& +R_{2}\left(\mathbf{K}_{2}\right)
\end{aligned}
$$

for some positive semidefinite matrices $\mathbf{K}_{0}, \mathbf{K}_{1}, \mathbf{K}_{2}$ such that $\operatorname{tr}\left(\mathbf{K}_{0}+\mathbf{K}_{1}+\mathbf{K}_{2}\right) \leq P$, and $\mathcal{R}_{2}^{\mathrm{DPC}}(P)$ can be obtained from $\mathcal{R}_{1}^{\mathrm{DPC}}(P)$ by swapping the subscripts 1 and 2 .

The DPC region is tight in several cases. The first one is the case where each receiver gets only a private message, i.e., $R_{0}=0$ [2]. The other case is the degraded message sets scenario in which we have either $R_{1}=0$ or $R_{2}=0$ [3]. In both of these cases, there are only two messages to be sent. The case when both private messages and a common message are present is investigated in [3] and [4]. In [3] and [4], outer bounds on the capacity region are given, and these outer bounds are shown to match the DPC region in certain regions. Furthermore, Weingarten et al. [3], [4] show that for a given common message rate $R_{0}$, the DPC region achieves the private message sum rate capacity, i.e., the maximum of $R_{1}+R_{2}$. Finally, they [3], [4] show that if the common message rate is beyond a certain threshold, the DPC region matches the capacity region if the channel input is subject to a covariance constraint, i.e., $E\left[\mathbf{X X}^{\top}\right] \preceq \mathbf{S}$ for some $\mathbf{S} \succeq \mathbf{0}$. However, this result cannot be extended to the case where the channel input is subject to a total power constraint [7]. In [8], we show that an outer bound for the capacity region can be obtained by extending the DPC region in the common message rate direction by a fixed amount. This fixed amount, i.e., the gap, depends on the available power $P$, and hence, is not necessarily finite.

\section{MAIN RESUlt}

We now present our main result. To this end, we introduce the GSVD [11], [13] which plays a crucial role in the proof of our main result, and provides the necessary notation to express the results.

Definition 1 ([11, Th. 1]): Given two matrices $\mathbf{H}_{1} \in \mathbb{R}^{r_{1} \times t}$ and $\mathbf{H}_{2} \in \mathbb{R}^{r_{2} \times t}$, there exist orthonormal matrices $\mathbf{\Psi}_{1} \in \mathbb{R}^{r_{1} \times r_{1}}, \mathbf{\Psi}_{2} \in \mathbb{R}^{r_{2} \times r_{2}}, \mathbf{\Psi}_{0} \in \mathbb{R}^{t \times t}$, a non-singular

${ }^{2}$ The DPC region $\mathcal{R}^{\mathrm{DPC}}(P)$ in [5] is stated in a different form; however the equivalence of the region in [5] and the one we use here can be shown by using equivalent descriptions of Marton's inner bound [12]. 
matrix $\boldsymbol{\Omega} \in \mathbb{R}^{k \times k}$, and two matrices $\boldsymbol{\Sigma}_{1} \in \mathbb{R}^{r_{1} \times k}, \boldsymbol{\Sigma}_{2} \in \mathbb{R}^{r_{2} \times k}$ such that

$$
\begin{aligned}
& \boldsymbol{\Psi}_{1}^{\top} \mathbf{H}_{1} \boldsymbol{\Psi}_{0}=\boldsymbol{\Sigma}_{1}\left[\boldsymbol{\Omega}^{-1} \mathbf{0}_{k \times t-k}\right] \\
& \boldsymbol{\Psi}_{2}^{\top} \mathbf{H}_{2} \boldsymbol{\Psi}_{0}=\boldsymbol{\Sigma}_{2}\left[\boldsymbol{\Omega}^{-1} \mathbf{0}_{k \times t-k}\right]
\end{aligned}
$$

where $\boldsymbol{\Sigma}_{1}$ and $\boldsymbol{\Sigma}_{2}$ are given by

$$
\begin{aligned}
& \boldsymbol{\Sigma}_{1}=\left[\begin{array}{lll}
\mathbf{I}_{k-p-s \times k-p-s} & & \\
& \mathbf{D}_{1, s \times s} & \\
& & \mathbf{0}_{r_{1}+p-k \times p}
\end{array}\right] \\
& \boldsymbol{\Sigma}_{2}=\left[\begin{array}{lll}
\mathbf{0}_{r_{2}-p-s \times k-p-s} & & \\
& \mathbf{D}_{2, s \times s} & \mathbf{I}_{p \times p}
\end{array}\right]
\end{aligned}
$$

and the constants $k, p$ are given as

$$
\begin{aligned}
& k=\operatorname{rank}\left(\left[\begin{array}{l}
\mathbf{H}_{1} \\
\mathbf{H}_{2}
\end{array}\right]\right) \\
& p=\operatorname{dim}\left(\operatorname{Null}\left(\mathbf{H}_{1}\right) \cap \operatorname{Null}\left(\mathbf{H}_{2}\right)^{\perp}\right)
\end{aligned}
$$

and $\mathrm{s}$ depends on the matrices $\mathbf{H}_{1}, \mathbf{H}_{2} . \mathbf{D}_{1}, \mathbf{D}_{2}$ are diagonal with the diagonal elements being strictly positive. $\Omega^{-1}$ can be expressed as $\boldsymbol{\Omega}^{-1}=\mathbf{W}^{\top} \mathbf{R}$, where $\mathbf{W}$ is an orthonormal matrix and $\mathbf{R}$ is a non-singular matrix whose singular values are given by the nonzero singular values of the following matrix:

$$
\left[\begin{array}{l}
\mathbf{H}_{1} \\
\mathbf{H}_{2}
\end{array}\right] \text {. }
$$

We define the sets $\mathcal{S}_{1}, \mathcal{S}_{c}, \mathcal{S}_{2}$ as follows:

$$
\begin{aligned}
& \mathcal{S}_{1}=\{1, \ldots, k-p-s\} \\
& \mathcal{S}_{c}=\{k-p-s+1, \ldots, k-p\} \\
& \mathcal{S}_{2}=\{k-p+1, \ldots, k\}
\end{aligned}
$$

respectively. We denote the nonzero entries of the matrices $\Sigma_{1}$ and $\boldsymbol{\Sigma}_{2}$ by

$$
\begin{array}{ll}
\tilde{h}_{1 \ell}=\Sigma_{1, \ell \ell}, & \ell \in \mathcal{S}_{1} \cup \mathcal{S}_{c} \\
\tilde{h}_{2 \ell}=\Sigma_{2,\left(r_{2}-k+\ell\right) \ell}, & \ell \in \mathcal{S}_{2} \cup \mathcal{S}_{c}
\end{array}
$$

respectively. Moreover, we partition the set $\mathcal{S}_{c}$ into two disjoint subsets $\mathcal{S}_{c 1}$ and $\mathcal{S}_{c 2}$ as follows:

$$
\begin{aligned}
& \mathcal{S}_{c 1}=\left\{\ell \in \mathcal{S}_{c}: \tilde{h}_{1 \ell}^{2} \geq \tilde{h}_{2 \ell}^{2}\right\} \\
& \mathcal{S}_{c 2}=\left\{\ell \in \mathcal{S}_{c}: \tilde{h}_{2 \ell}^{2} \geq \tilde{h}_{1 \ell}^{2}\right\} .
\end{aligned}
$$

We denote the maximum singular value of $\mathbf{R}$ by $\lambda_{\max }$ (which is also the maximum singular value of the matrix given in (17)), and the minimum singular value of $\mathbf{R}$ by $\lambda_{\min }$ (which is also the minimum nonzero singular value of the matrix given in (17)). Finally, we define the function $C(x)=(1 / 2) \log (1+$ $x), \forall x \geq 0$.

Next, we present our outer bound for the capacity region of the Gaussian MIMO broadcast channel with common and privates messages as follows.
Theorem 2: The capacity region of the Gaussian MIMO broadcast channel with common and private messages is contained in the region $\mathcal{R}^{\text {out }}(P)$ which is given by the union of rate triples $\left(R_{0}, R_{1}, R_{2}\right)$ satisfying

$$
\begin{aligned}
R_{0}+R_{1} \leq & \sum_{\ell \in \mathcal{S}_{c 2}} C\left(\frac{\tilde{h}_{1 \ell}^{2} \gamma_{\ell} P_{\ell}}{1+\tilde{h}_{1 \ell}^{2} \bar{\gamma}_{\ell} P_{\ell}}\right)+\sum_{\ell \in \mathcal{S}_{1} \cup \mathcal{S}_{c 1}} C\left(\tilde{h}_{1 \ell}^{2} P_{\ell}\right)(25) \\
R_{0}+R_{2} \leq & \sum_{\ell \in \mathcal{S}_{c 1}} C\left(\frac{\tilde{h}_{2 \ell}^{2} \gamma_{\ell} P_{\ell}}{1+\tilde{h}_{2 \ell}^{2} \bar{\gamma}_{\ell} P_{\ell}}\right)+\sum_{\ell \in \mathcal{S}_{2} \cup \mathcal{S}_{c 2}} C\left(\tilde{h}_{2 \ell}^{2} P_{\ell}\right)(26) \\
\sum_{j=0}^{2} R_{j} \leq & \sum_{\ell \in \mathcal{S}_{c 2}} C\left(\frac{\tilde{h}_{1 \ell}^{2} \gamma_{\ell} P_{\ell}}{1+\tilde{h}_{1 \ell}^{2} \bar{\gamma}_{\ell} P_{\ell}}\right)+\sum_{\ell \in \mathcal{S}_{2} \cup \mathcal{S}_{c 2}} C\left(\tilde{h}_{2 \ell}^{2} \bar{\gamma}_{\ell} P_{\ell}\right) \\
& +\sum_{\ell \in \mathcal{S}_{1} \cup \mathcal{S}_{c 1}} C\left(\tilde{h}_{1 \ell}^{2} P_{\ell}\right) \\
\sum_{j=0}^{2} R_{j} \leq & \sum_{\ell \in \mathcal{S}_{c 1}} C\left(\frac{\tilde{h}_{2 \ell}^{2} \gamma_{\ell} P_{\ell}}{1+\tilde{h}_{2 \ell}^{2} \bar{\gamma}_{\ell} P_{\ell}}\right)+\sum_{\ell \in \mathcal{S}_{1} \cup \mathcal{S}_{c 1}} C\left(\tilde{h}_{1 \ell}^{2} \bar{\gamma}_{\ell} P_{\ell}\right) \\
& +\sum_{\ell \in \mathcal{S}_{2} \cup \mathcal{S}_{c 2}} C\left(\tilde{h}_{2 \ell}^{2} P_{\ell}\right)
\end{aligned}
$$

for some $\gamma_{\ell}=1-\bar{\gamma}_{\ell} \in[0,1], \ell=1, \ldots,\left|\mathcal{S}_{1}\right|+\left|\mathcal{S}_{c}\right|+\left|\mathcal{S}_{2}\right|$, and $\left\{P_{\ell}\right\}_{\ell=1}^{\left|\mathcal{S}_{1}\right|+\left|\mathcal{S}_{c}\right|+\left|\mathcal{S}_{2}\right|}$ such that

$$
\sum_{\ell=1}^{\left|\mathcal{S}_{1}\right|+\left|\mathcal{S}_{c}\right|+\left|\mathcal{S}_{2}\right|} P_{\ell}=\lambda_{\max }^{2} P .
$$

The proof of Theorem 2 is given in Section IV. We obtain the outer bound in Theorem 2 in two steps. In the first step, we use the GSVD on the channel gain matrices $\mathbf{H}_{1}, \mathbf{H}_{2}$ and apply a relaxation on the power constraint to obtain a parallel Gaussian broadcast channel whose capacity region includes the capacity region of the original Gaussian MIMO broadcast channel. In the second step, we obtain the capacity region of this parallel Gaussian broadcast channel which gives us the outer bound in Theorem 2.

Now, we obtain an inner bound for the capacity region as stated in the following theorem.

Theorem 3: An inner bound for the capacity region of the Gaussian MIMO broadcast channel with common and private messages is given by the region $\mathcal{R}^{i n}(P)$ which contains all rate triples $\left(R_{0}, R_{1}, R_{2}\right)$ satisfying

$$
\begin{aligned}
R_{0}+R_{1} \leq & \sum_{\ell \in \mathcal{S}_{c 2}} C\left(\frac{\tilde{h}_{1 \ell}^{2} \gamma_{\ell} P_{\ell}}{1+\tilde{h}_{1 \ell}^{2} \bar{\gamma}_{\ell} P_{\ell}}\right)+\sum_{\ell \in \mathcal{S}_{1} \cup \mathcal{S}_{c 1}} C\left(\tilde{h}_{1 \ell}^{2} P_{\ell}\right) \\
R_{0}+R_{2} \leq & \sum_{\ell \in \mathcal{S}_{c 1}} C\left(\frac{\tilde{h}_{2 \ell}^{2} \gamma_{\ell} P_{\ell}}{1+\tilde{h}_{2 \ell}^{2} \bar{\gamma}_{\ell} P_{\ell}}\right)+\sum_{\ell \in \mathcal{S}_{2} \cup \mathcal{S}_{c 2}} C\left(\tilde{h}_{2 \ell}^{2} P_{\ell}\right) \\
\sum_{j=0}^{2} R_{j} \leq & \sum_{\ell \in \mathcal{S}_{c 2}} C\left(\frac{\tilde{h}_{1 \ell}^{2} \gamma_{\ell} P_{\ell}}{1+\tilde{h}_{1 \ell}^{2} \bar{\gamma}_{\ell} P_{\ell}}\right)+\sum_{\ell \in \mathcal{S}_{2} \cup \mathcal{S}_{c 2}} C\left(\tilde{h}_{2 \ell}^{2} \bar{\gamma}_{\ell} P_{\ell}\right) \\
& +\sum_{\ell \in \mathcal{S}_{1} \cup \mathcal{S}_{c 1}} C\left(\tilde{h}_{1 \ell}^{2} P_{\ell}\right) \\
\sum_{j=0}^{2} R_{j} \leq & \sum_{\ell \in \mathcal{S}_{c 1}} C\left(\frac{\tilde{h}_{2 \ell}^{2} \gamma_{\ell} P_{\ell}}{1+\tilde{h}_{2 \ell}^{2} \bar{\gamma}_{\ell} P_{\ell}}\right)+\sum_{\ell \in \mathcal{S}_{1} \cup \mathcal{S}_{c 1}} C\left(\tilde{h}_{1 \ell}^{2} \bar{\gamma}_{\ell} P_{\ell}\right) \\
& +\sum_{\ell \in \mathcal{S}_{2} \cup \mathcal{S}_{c 2}} C\left(\tilde{h}_{2 \ell}^{2} P_{\ell}\right)
\end{aligned}
$$


for some $\gamma_{\ell}=1-\bar{\gamma}_{\ell} \in[0,1], \ell=1, \ldots,\left|\mathcal{S}_{1}\right|+\left|\mathcal{S}_{c}\right|+\left|\mathcal{S}_{2}\right|$, and $\left\{P_{\ell}\right\}_{\ell=1}^{\left|\mathcal{S}_{1}\right|+\left|\mathcal{S}_{c}\right|+\left|\mathcal{S}_{2}\right|}$ such that

$$
\sum_{\ell=1}^{\left|\mathcal{S}_{1}\right|+\left|\mathcal{S}_{c}\right|+\left|\mathcal{S}_{2}\right|} P_{\ell}=\lambda_{\min }^{2} P .
$$

The proof of Theorem 3 is given in Section V. We obtain the inner bound in Theorem 3 in two different ways. In the first way, we choose the covariance matrices $\mathbf{K}_{0}, \mathbf{K}_{1}, \mathbf{K}_{2}$ involved in the DPC region given by Theorem 1 in a specific form. Since this specific choice of the covariance matrices is not necessarily optimal, the inner bound in Theorem 3 is potentially looser than the DPC region given by Theorem 1 . However, the inner bound in Theorem 3 is more readily amenable for a comparison with the outer bound in Theorem 2 . In the second way, we use a variation of the ZF scheme to obtain the inner bound in Theorem 3 . The difference of our ZF scheme from the standard ZF scheme is that while the standard $\mathrm{ZF}$ scheme uses individual singular value decompositions to determine the transmit directions, our ZF scheme uses the GSVD to determine the transmit directions.

We note that the only difference between the outer bound in Theorem 2 and the inner bound in Theorem 3 is the power constraints, see (29) and (34). In fact, this observation implies that the outer bound in Theorem 2 and the inner bound in Theorem 3 can characterize the capacity region with a finite gap, i.e., the gap between the boundaries of the outer bound and the inner bound does not scale with the available power $P$, as stated in the following theorem.

Theorem 4: If $\left(R_{0}, R_{1}, R_{2}\right)$ is a rate tuple on the boundary of the inner bound $\mathcal{R}^{i n}(P)$, then $\left(R_{0}+\alpha_{0}, R_{1}+\alpha_{1}, R_{2}+\alpha_{2}\right)$ is not achievable for any nonnegative $\left(\alpha_{0}, \alpha_{1}, \alpha_{2}\right)$ satisfying

$$
\begin{array}{r}
\left(\left|\mathcal{S}_{1}\right|+\left|\mathcal{S}_{c}\right|\right) \log \frac{\lambda_{\max }}{\lambda_{\min }} \leq \alpha_{0}+\alpha_{1} \\
\left(\left|\mathcal{S}_{2}\right|+\left|\mathcal{S}_{c}\right|\right) \log \frac{\lambda_{\max }}{\lambda_{\min }} \leq \alpha_{0}+\alpha_{2} \\
\left(\max _{j=1,2}\left|\mathcal{S}_{c j}\right|+\left|\mathcal{S}_{1}\right|+\left|\mathcal{S}_{c}\right|+\left|\mathcal{S}_{2}\right|\right) \log \frac{\lambda_{\max }}{\lambda_{\min }} \leq \alpha_{0}+\alpha_{1}+\alpha_{2} .
\end{array}
$$

The proof of Theorem 4 is given in Appendix A. Since there is a finite gap between the outer bound in Theorem 2 and the inner bound in Theorem 3 as stated by Theorem 4, we can also characterize the DoF region ${ }^{3}$ of the Gaussian MIMO broadcast channel with common and private messages as follows.

Theorem 5: The DoF region of the Gaussian MIMO broadcast channel with common and private messages is given by the union of DoF triples $\left(d_{0}, d_{1}, d_{2}\right)$ satisfying

$$
\begin{aligned}
d_{0}+d_{1} & \leq\left|\mathcal{S}_{1}\right|+\left|\mathcal{S}_{c}\right| \\
d_{0}+d_{2} & \leq\left|\mathcal{S}_{2}\right|+\left|\mathcal{S}_{c}\right| \\
d_{0}+d_{1}+d_{2} & \leq\left|\mathcal{S}_{1}\right|+\left|\mathcal{S}_{c}\right|+\left|\mathcal{S}_{2}\right| .
\end{aligned}
$$

${ }^{3} \mathrm{~A}$ DoF triple $\left(d_{0}, d_{1}, d_{2}\right)$ is said to be achievable if there exists a rate triple $\left(R_{0}, R_{1}, R_{2}\right) \in \mathcal{C}(P)$ such that $d_{j}=\lim _{P \rightarrow \infty}\left(2 R_{j} / \log P\right), j=0,1,2$. The DoF region is defined as the convex closure of all achievable DoF triples $\left(d_{0}, d_{1}, d_{2}\right)$.
Theorem 5 can be proved either by using the outer bound in Theorem 2 or by using the inner bound in Theorem 3 . Theorem 5 states that for sufficiently large power $P$, the Gaussian MIMO broadcast channel is equivalent to a parallel Gaussian broadcast channel with $\left|\mathcal{S}_{1}\right|+\left|\mathcal{S}_{c}\right|+\left|\mathcal{S}_{2}\right|$ subchannels, where $\left|\mathcal{S}_{j}\right|$ of these subchannels can be accessed by only the $j$ th user and $\left|\mathcal{S}_{c}\right|$ of these subchannels can be accessed by both users. This equivalence arises from the facts that when we apply the GSVD to the Gaussian MIMO broadcast channel, it becomes a Gaussian MIMO broadcast channel with $\left|\mathcal{S}_{1}\right|+\left|\mathcal{S}_{c}\right|+\left|\mathcal{S}_{2}\right|$ transmit antennas, and $\left|\mathcal{S}_{j}\right|+\left|\mathcal{S}_{c}\right|$ receive antennas at the $j$ th user, and for sufficiently large power, the Gaussian MIMO broadcast channel reduces a parallel Gaussian broadcast channel.

\section{Proof of Theorem 2}

Here, we prove Theorem 2 in two steps. In the first step, we obtain a parallel Gaussian broadcast channel from the original channel in (1)-(3) by using the GSVD and a relaxation on the power constraint. Due this power relaxation, the capacity region of the parallel Gaussian broadcast channel provides an outer bound for the capacity region of the original channel. In the second step, we use the capacity result for the parallel Gaussian broadcast channel given in [6] to complete the proof.

First, we note that since $\boldsymbol{\Psi}_{j}$ in (11)-(12) is orthonormal, i.e., non-singular, multiplying the channel output $\mathbf{Y}_{j}$ by $\boldsymbol{\Psi}_{j}^{\top}$ does not alter the capacity region. Hence, the following channel

$$
\begin{aligned}
\tilde{\mathbf{Y}}_{j} & =\boldsymbol{\Psi}_{j}^{\top} \mathbf{H}_{j} \mathbf{X}+\mathbf{\Psi}_{j}^{\top} \mathbf{N}_{j} \\
& =\boldsymbol{\Sigma}_{j}\left[\begin{array}{ll}
\boldsymbol{\Omega}^{-1} & \mathbf{0}_{k \times t-k}
\end{array}\right] \mathbf{\Psi}_{0}^{\top} \mathbf{X}+\mathbf{\Psi}_{j}^{\top} \mathbf{N}_{j}, \quad j=1,2(42)
\end{aligned}
$$

has the same capacity region with the original channel in (1)-(3), where (42) is obtained by using (11)-(12). We define $\tilde{\mathbf{N}}_{j}=\boldsymbol{\Psi}_{j}^{\top} \mathbf{N}_{j}$ which is also a white Gaussian random vector, i.e., $E\left[\tilde{\mathbf{N}}_{j} \tilde{\mathbf{N}}_{j}^{\top}\right]=\mathbf{I}$, due to the fact that $\mathbf{\Psi}_{j}$ is orthonormal and $\mathbf{N}_{j}$ is white. We also define

$$
\tilde{\mathbf{X}}=\left[\begin{array}{ll}
\boldsymbol{\Omega}^{-1} & \mathbf{0}_{k \times t-k}
\end{array}\right] \mathbf{\Psi}_{0}^{\top} \mathbf{X} .
$$

Next, we note that the last $r_{1}+p-k$ entries of $\tilde{\mathbf{Y}}_{1}$ and the first $r_{2}-p-s$ entries of $\tilde{\mathbf{Y}}_{2}$ come from only the noise. Since both $\tilde{\mathbf{N}}_{1}$ and $\tilde{\mathbf{N}}_{2}$ are white, we can omit the last $r_{1}+p-k$ entries of $\tilde{\mathbf{Y}}_{1}$ and the first $r_{2}-p-s$ entries of $\tilde{\mathbf{Y}}_{2}$ without loss of generality. Using the definitions in (21)-(22) and omitting the entries of $\tilde{\mathbf{Y}}_{1}, \tilde{\mathbf{Y}}_{2}$ which contain only noise, the channel in (42) can be expressed as

$$
\begin{aligned}
& \tilde{Y}_{1 \ell}=\tilde{h}_{1 \ell} \tilde{X}_{\ell}+\tilde{N}_{1 \ell}, \quad \ell \in \mathcal{S}_{1} \cup \mathcal{S}_{c} \\
& \tilde{Y}_{2 \ell}=\tilde{h}_{2 \ell} \tilde{X}_{\ell}+\tilde{N}_{2 \ell}, \quad \ell \in \mathcal{S}_{2} \cup \mathcal{S}_{c}
\end{aligned}
$$

where we used the definitions of $\mathcal{S}_{1}, \mathcal{S}_{c}, \mathcal{S}_{2}$ given in (18)-(20), and $\left\{\tilde{N}_{1, \ell}\right\}_{\ell \in \mathcal{S}_{1} \cup \mathcal{S}_{c}},\left\{\tilde{N}_{2, \ell}\right\}_{\ell \in \mathcal{S}_{1} \cup \mathcal{S}_{c}}$ are i.i.d. Gaussian random variables with unit variance. 
Now, we relax the power constraint on $\tilde{\mathbf{X}}$, and consequently, obtain a new channel whose capacity region includes the capacity region of the original channel in (1)-(2)-(3). To this end, we note that

$$
\begin{aligned}
& \sum_{\ell=1}^{\left|\mathcal{S}_{1}\right|+\left|\mathcal{S}_{c}\right|+\left|\mathcal{S}_{2}\right|} E\left[\tilde{X}_{\ell}^{2}\right]=\operatorname{tr}\left(E\left[\tilde{\mathbf{X}} \tilde{\mathbf{X}}^{\top}\right]\right) \\
= & \operatorname{tr}\left(\left[\boldsymbol{\Omega}^{-1} \mathbf{0}_{k \times t-k}\right] \mathbf{\Psi}_{0}^{\top} E\left[\mathbf{X} \mathbf{X}^{\top}\right] \mathbf{\Psi}_{0}\left[\boldsymbol{\Omega}^{-1} \mathbf{0}_{k \times t-k}\right]^{\top}\right) \\
= & \operatorname{tr}\left(\boldsymbol{\Psi}_{0}^{\top} E\left[\mathbf{X X}^{\top}\right] \mathbf{\Psi}_{0}\left[\boldsymbol{\Omega}^{-1} \mathbf{0}_{k \times t-k}\right]^{\top}\left[\boldsymbol{\Omega}^{-1} \mathbf{0}_{k \times t-k}\right]\right)
\end{aligned}
$$

where (47) comes from the definition of $\tilde{\mathbf{X}}$ in (43), and (48) comes from the fact that $\operatorname{tr}(\mathbf{A B})=\operatorname{tr}(\mathbf{B A})$. Next, we note that

$$
\begin{aligned}
\mathbf{0} & \preceq\left[\boldsymbol{\Omega}^{-1} \mathbf{0}_{k \times t-k}\right]^{\top}\left[\boldsymbol{\Omega}^{-1} \mathbf{0}_{k \times t-k}\right] \\
& \preceq \sigma_{\max }\left(\left(\boldsymbol{\Omega}^{-1}\right)^{\top} \boldsymbol{\Omega}^{-1}\right) \mathbf{I} \\
& =\sigma_{\max }\left(\mathbf{R}^{\top} \mathbf{R}\right) \mathbf{I} \\
& =\sigma_{\max }^{2}(\mathbf{R}) \mathbf{I} \\
& =\lambda_{\max }^{2} \mathbf{I}
\end{aligned}
$$

where $\sigma_{\max }(\cdot)$ denotes the maximum singular value of the corresponding matrix, in (51), we use $\boldsymbol{\Omega}^{-1}=\mathbf{W}^{\top} \mathbf{R}$ and the fact that $\mathbf{W}$ is an orthonormal matrix, and (53) comes from the definition of $\lambda_{\text {max }}$. Since $\operatorname{tr}(\mathbf{A B}) \geq 0$ if $\mathbf{A} \succeq \mathbf{0}, \mathbf{B} \succeq \mathbf{0}$, using (53) in (48), we get

$$
\begin{aligned}
\sum_{\ell=1}^{\mathcal{S}_{1}|+| \mathcal{S}_{c}|+| \mathcal{S}_{2} \mid} E\left[\tilde{X}_{\ell}^{2}\right] & \leq \lambda_{\max }^{2} \operatorname{tr}\left(\mathbf{\Psi}_{0}^{\top} E\left[\mathbf{X} \mathbf{X}^{\top}\right] \mathbf{\Psi}_{0}\right) \\
& =\lambda_{\max }^{2} \operatorname{tr}\left(E\left[\mathbf{X} \mathbf{X}^{\top}\right] \mathbf{\Psi}_{0} \mathbf{\Psi}_{0}^{\top}\right) \\
& =\lambda_{\max }^{2}\left(E\left[\mathbf{X} \mathbf{X}^{\top}\right]\right) \\
& \leq \lambda_{\max }^{2} P
\end{aligned}
$$

where (55) is due to the fact that $\operatorname{tr}(\mathbf{A B})=\operatorname{tr}(\mathbf{B A}),(56)$ comes from the fact that $\mathbf{\Psi}_{0}$ is orthonormal, and (57) is due to the total power constraint on $\mathbf{X}$ given in (3). Hence, we obtain the following channel:

$$
\begin{array}{ll}
\tilde{Y}_{1 \ell}=\tilde{h}_{1 \ell} \tilde{X}_{\ell}+\tilde{N}_{1 \ell}, & \ell \in \mathcal{S}_{1} \cup \mathcal{S}_{c} \\
\tilde{Y}_{2 \ell}=\tilde{h}_{2 \ell} \tilde{X}_{\ell}+\tilde{N}_{2 \ell}, & \ell \in \mathcal{S}_{2} \cup \mathcal{S}_{c}
\end{array}
$$

where the channel input is subject to the following constraint:

$$
\sum_{\ell=1}^{\left|\mathcal{S}_{1}\right|+\left|\mathcal{S}_{c}\right|+\left|\mathcal{S}_{2}\right|} E\left[\tilde{X}_{\ell}^{2}\right] \leq \lambda_{\max }^{2} P .
$$

We note that this new channel in (58)-(60) is obtained from the original channel in (1)-(3) by two operations: The first one is the multiplication of the channel outputs in the original channel, with invertible matrices $\boldsymbol{\Psi}_{1}, \boldsymbol{\Psi}_{2}$, which preserves the capacity region. The second operation is the relaxation of the power constraint (cf., (50) and (54)) in the new channel to get (60) which enlarges the capacity region by means of increasing the set of all feasible input distributions. Thus, due to this second operation, the capacity region of the new channel in (58)-(60) serves as an outer bound for the capacity region of the original channel in (1)-(3). Moreover, the channel defined by (58)-(60) is a parallel Gaussian broadcast channel, whose capacity region is obtained in [6]. Hence, using this capacity result, one can obtain the region given in Theorem 2, completing the proof.

\section{Proof of Theorem 3}

In this section, we provide the proof of the inner bound for the capacity region given in Theorem 3 . In particular, we provide two different achievable schemes for the region given in Theorem 3. The first one, presented in Section V-A, uses the DPC region in Theorem 1 directly. The second one, presented in Section V-B, uses a variation of the ZF scheme [14], [15].

\section{A. DPC-Based Achievable Scheme}

We now obtain the inner bound given by Theorem 3 by using the DPC region in Theorem 1. To this end, we choose the covariance matrices $\left\{\mathbf{K}_{u}\right\}_{u=0}^{2}$ involved in the DPC region given by Theorem 3 as follows

$$
\mathbf{K}_{u}=\mathbf{\Psi}_{0}\left[\boldsymbol{\Omega}^{\top} \mathbf{0}_{k \times t-k}\right]^{\top} \boldsymbol{\Lambda}_{u}\left[\boldsymbol{\Omega}^{\top} \mathbf{0}_{k \times t-k}\right] \mathbf{\Psi}_{0}^{\top}
$$

where $\boldsymbol{\Lambda}_{u}$ is a diagonal matrix of size $k \times k$, and we have the power constraint $\operatorname{tr}\left(\mathbf{K}_{0}+\mathbf{K}_{1}+\mathbf{K}_{2}\right) \leq P$. Moreover, we choose the diagonal matrices $\boldsymbol{\Lambda}_{0}, \boldsymbol{\Lambda}_{1}, \boldsymbol{\Lambda}_{2}$ as follows

$$
\begin{aligned}
\Lambda_{0, \ell \ell}+\Lambda_{1, \ell \ell}+\Lambda_{2, \ell \ell} & =P_{\ell} & & \forall \ell \in \mathcal{S}_{1} \cup \mathcal{S}_{2} \cup \mathcal{S}_{c} \\
\Lambda_{0, \ell \ell} & =\gamma_{\ell} P_{\ell} & & \forall \ell \in \mathcal{S}_{1} \cup \mathcal{S}_{2} \cup \mathcal{S}_{c} \\
\Lambda_{1, \ell \ell} & =0 & & \forall \ell \in \mathcal{S}_{2} \cup \mathcal{S}_{c 2} \\
\Lambda_{2, \ell \ell} & =0 & & \forall \ell \in \mathcal{S}_{1} \cup \mathcal{S}_{c 1} .
\end{aligned}
$$

Next, we note the following identity

$$
\mathbf{H}_{j} \mathbf{K}_{u} \mathbf{H}_{j}^{\top}=\boldsymbol{\Psi}_{j} \boldsymbol{\Sigma}_{j} \boldsymbol{\Lambda}_{u} \boldsymbol{\Sigma}_{j}^{\top} \boldsymbol{\Psi}_{j}^{\top}, \quad j=1,2, u=0,1,2
$$

which can be obtained by using (61) and the identity $\mathbf{H}_{j}=\boldsymbol{\Psi}_{j} \boldsymbol{\Sigma}_{j}\left[\boldsymbol{\Omega}^{-1} \mathbf{0}_{k \times t-k}\right] \mathbf{\Psi}_{0}^{\top}$ which is a consequence of the GSVD in (11)-(12). Thus, using the covariance matrices $\mathbf{K}_{0}, \mathbf{K}_{1}, \mathbf{K}_{2}$ defined by (61) for the rate expressions in (4)-(6) and the identity in (66), we get

$R_{0 j}\left(\mathbf{K}_{0}, \mathbf{K}_{1}, \mathbf{K}_{2}\right)=\frac{1}{2} \log \frac{\left|\boldsymbol{\Psi}_{j} \boldsymbol{\Sigma}_{j}\left(\boldsymbol{\Lambda}_{0}+\boldsymbol{\Lambda}_{1}+\boldsymbol{\Lambda}_{2}\right) \boldsymbol{\Sigma}_{j}^{\top} \mathbf{\Psi}_{j}^{\top}+\mathbf{I}\right|}{\left|\boldsymbol{\Psi}_{j} \boldsymbol{\Sigma}_{j}\left(\boldsymbol{\Lambda}_{1}+\boldsymbol{\Lambda}_{2}\right) \boldsymbol{\Sigma}_{j}^{\top} \mathbf{\Psi}_{j}^{\top}+\mathbf{I}\right|}$

$$
\begin{aligned}
R_{1}\left(\mathbf{K}_{1}, \mathbf{K}_{2}\right) & =\frac{1}{2} \log \frac{\left|\boldsymbol{\Psi}_{1} \boldsymbol{\Sigma}_{1}\left(\boldsymbol{\Lambda}_{1}+\boldsymbol{\Lambda}_{2}\right) \boldsymbol{\Sigma}_{1}^{\top} \boldsymbol{\Psi}_{1}^{\top}+\mathbf{I}\right|}{\left|\mathbf{\Psi}_{1} \boldsymbol{\Sigma}_{1} \boldsymbol{\Lambda}_{2} \boldsymbol{\Sigma}_{1}^{\top} \mathbf{\Psi}_{1}^{\top}+\mathbf{I}\right|} \\
R_{2}\left(\mathbf{K}_{2}\right) & =\frac{1}{2} \log \left|\boldsymbol{\Psi}_{2} \boldsymbol{\Sigma}_{2} \boldsymbol{\Lambda}_{2} \boldsymbol{\Sigma}_{2}^{\top} \boldsymbol{\Psi}_{2}^{\top}+\mathbf{I}\right| .
\end{aligned}
$$

Using Slyvester's determinant theorem, i.e., $\mid \mathbf{A}_{m \times n} \mathbf{B}_{n \times m}+$ $\mathbf{I}_{m \times m}|=| \mathbf{B}_{n \times m} \mathbf{A}_{m \times n}+\mathbf{I}_{n \times n} \mid$, the diagonal structure of the matrices $\boldsymbol{\Sigma}_{j}^{\top} \boldsymbol{\Sigma}_{j}$ and the specific form of the diagonal matrices 
$\boldsymbol{\Lambda}_{0}, \boldsymbol{\Lambda}_{1}, \boldsymbol{\Lambda}_{2}$ given in (62)-(65), (67)-(69) can be expressed as follows

$$
\begin{aligned}
R_{0 j}\left(\mathbf{K}_{0}, \mathbf{K}_{1}, \mathbf{K}_{2}\right) & =\sum_{\ell \in \mathcal{S}_{j} \cup \mathcal{S}_{c}} \frac{1}{2} \log \left(1+\frac{\tilde{h}_{j \ell}^{2} \gamma_{\ell} P_{\ell}}{1+\tilde{h}_{j \ell}^{2} \bar{\gamma}_{\ell} P_{\ell}}\right) \\
R_{1}\left(\mathbf{K}_{1}, \mathbf{K}_{2}\right) & =\sum_{\ell \in \mathcal{S}_{1} \cup \mathcal{S}_{c 1}} \frac{1}{2} \log \left(1+\tilde{h}_{1 \ell}^{2} \bar{\gamma}_{\ell} P_{\ell}\right) \\
R_{2}\left(\mathbf{K}_{2}\right) & =\sum_{\ell \in \mathcal{S}_{2} \cup \mathcal{S}_{c 2}} \frac{1}{2} \log \left(1+\tilde{h}_{2 \ell}^{2} \bar{\gamma}_{\ell} P_{\ell}\right)
\end{aligned}
$$

using which in the rate bounds in Theorem 1, one can obtain the rate region given in Theorem 3 .

We are left only with the proof of the power constraint in (34). Next, we determine this constraint. To this end, we consider

$$
\begin{array}{r}
\operatorname{tr}\left(\mathbf{K}_{0}+\mathbf{K}_{1}+\mathbf{K}_{2}\right)=\operatorname{tr}\left(\left(\boldsymbol{\Lambda}_{0}+\boldsymbol{\Lambda}_{1}+\boldsymbol{\Lambda}_{2}\right)\left(\boldsymbol{\Omega}^{\top} \boldsymbol{\Omega}\right)\right) \\
=\operatorname{tr}\left(\left(\boldsymbol{\Lambda}_{0}+\boldsymbol{\Lambda}_{1}+\boldsymbol{\Lambda}_{2}\right) \mathbf{W}^{\top}\left(\mathbf{R} \mathbf{R}^{\top}\right)^{-1} \mathbf{W}\right)
\end{array}
$$

where (73) comes from the definition of $\mathbf{K}_{0}, \mathbf{K}_{1}, \mathbf{K}_{2}$ in (61) and the fact that $\operatorname{tr}(\mathbf{A B})=\operatorname{tr}(\mathbf{B A})$, and (74) follows from the definition of $\boldsymbol{\Omega}$, i.e., $\boldsymbol{\Omega}^{-1}=\mathbf{W}^{\top} \mathbf{R}$. Next, we note that

$$
\mathbf{R R}^{\top} \succeq \lambda_{\min }^{2} \mathbf{I}
$$

which implies

$$
\left(\mathbf{R R}^{\top}\right)^{-1} \preceq \frac{1}{\lambda_{\min }^{2}} \mathbf{I}
$$

using which and the fact that $\operatorname{tr}(\mathbf{A B}) \geq 0$ for $\mathbf{A} \succeq \mathbf{0}, \mathbf{B} \succeq \mathbf{0}$ in (74), we get

$$
\begin{aligned}
\operatorname{tr}\left(\mathbf{K}_{0}+\mathbf{K}_{1}+\mathbf{K}_{2}\right) & \left.\leq \frac{1}{\lambda_{\text {min }}^{2}} \operatorname{tr}\left(\boldsymbol{\Lambda}_{0}+\boldsymbol{\Lambda}_{1}+\boldsymbol{\Lambda}_{2}\right) \mathbf{W}^{\top} \mathbf{W}\right) \\
& =\frac{1}{\lambda_{\text {min }}^{2}} \operatorname{tr}\left(\boldsymbol{\Lambda}_{0}+\boldsymbol{\Lambda}_{1}+\mathbf{\Lambda}_{2}\right) \\
& =\frac{1}{\lambda_{\text {min }}^{2}} \sum_{\ell=1}^{\left|\mathcal{S}_{1}\right|+\left|\mathcal{S}_{2}\right|+\left|\mathcal{S}_{c}\right|} P_{\ell}
\end{aligned}
$$

where (78) follows from the orthonormality of $\mathbf{W}$ and (79) comes from (62). Equation (79) implies that if the constraint in (34) is satisfied, then the covariance matrices $\mathbf{K}_{0}, \mathbf{K}_{1}, \mathbf{K}_{2}$ satisfy the power constraint on them; completing the proof.

\section{B. ZF-Based Achievable Scheme}

In this section, we show that a variation of the ZF scheme also attains the inner bound given in Theorem 3 . The original form of the ZF scheme is proposed for the Gaussian MIMO broadcast channel with only private messages, i.e., without a common message, where the transmitter sends the private message of each user through the null space of the other user. Since this original ZF scheme considers the individual singular value decompositions of the channel gain matrices, it has some restrictions as it can attain the DoF of the private message sum rate ${ }^{4}$ only when $r_{1}+r_{2} \leq t$ [14], [15]. However, by using the GSVD of the two channel gain matrices simultaneously, this restriction can be removed as we do here. In the variation of the ZF scheme we propose here, the transmitter sends

$$
\mathbf{X}=\mathbf{\Psi}_{0}\left[\boldsymbol{\Omega}^{\top} \mathbf{0}_{k \times t-k}\right]^{\top} \tilde{\mathbf{X}}
$$

Consequently, the received signal at the $j$ th user can be written as

$$
\begin{aligned}
\mathbf{Y}_{j} & =\mathbf{H}_{j} \mathbf{X}+\mathbf{N}_{j} \\
& =\boldsymbol{\Psi}_{j} \boldsymbol{\Sigma}_{j} \tilde{\mathbf{X}}+\mathbf{N}_{j}
\end{aligned}
$$

where (82) is a consequence of the GSVD and (80). After multiplying $\mathbf{Y}_{j}$ by the orthonormal matrix $\boldsymbol{\Psi}_{j}^{\top}$, we get

$$
\begin{aligned}
& \hat{\mathbf{Y}}_{1}=\boldsymbol{\Sigma}_{1} \tilde{\mathbf{X}}+\hat{\mathbf{N}}_{1} \\
& \hat{\mathbf{Y}}_{2}=\boldsymbol{\Sigma}_{2} \tilde{\mathbf{X}}+\hat{\mathbf{N}}_{2}
\end{aligned}
$$

where $\hat{\mathbf{N}}_{j}=\mathbf{\Psi}_{j}^{\top} \mathbf{N}_{j}$ is a Gaussian vector with identity covariance matrix. This equivalent form of the channel in (83)-(84), which results from the use of the ZF scheme, implies that, since $\boldsymbol{\Sigma}_{1}$ and $\boldsymbol{\Sigma}_{2}$ are diagonal, the ZF scheme transforms the channel into a parallel Gaussian broadcast channel. In fact, this channel model is the one we used in Section IV to obtain the outer bound for the capacity region in Theorem 2. In Section IV, we first relaxed the power constraint to obtain a channel whose capacity region is larger than the original one, and then, used the capacity result from [6] to obtain the outer bound in Theorem 2. Here, instead, we will further strengthen the power constraint and use the capacity result from [6] to obtain the inner bound in Theorem 3 . We note that the trace of the input in (80) is

$$
\operatorname{tr}\left(E\left[\mathbf{X X}^{\top}\right]\right)=\operatorname{tr}\left(E\left[\tilde{\mathbf{X}} \tilde{\mathbf{X}}^{\top}\right]\left(\boldsymbol{\Omega}^{\top} \boldsymbol{\Omega}\right)\right)
$$

where (85) comes from the definition of $\mathbf{X}$ in (80) and the fact that $\operatorname{tr}(\mathbf{A B})=\operatorname{tr}(\mathbf{B A})$. We note that (85) is similar to (73), and hence, can be bounded similarly leading to

$$
\operatorname{tr}\left(E\left[\tilde{\mathbf{X}} \tilde{\mathbf{X}}^{\top}\right]\left(\boldsymbol{\Omega}^{\top} \boldsymbol{\Omega}\right)\right) \leq \frac{1}{\lambda_{\min }^{2}} \sum_{\ell \in \mathcal{S}_{1} \cup \mathcal{S}_{c} \in \mathcal{S}_{2}} E\left[\tilde{X}_{\ell}^{2}\right]
$$

using which, we can strengthen the power constraint as follows

$$
\frac{1}{\lambda_{\min }^{2}} \sum_{\ell \in \mathcal{S}_{1} \cup \mathcal{S}_{c} \in \mathcal{S}_{2}} E\left[\tilde{X}_{\ell}^{2}\right] \leq P .
$$

Thus, we obtain a parallel Gaussian broadcast channel given by (83)-(84) and (87) for which the achievability of the region given in Theorem 3 follows from [6].

\section{CONCLUSION}

Here, we consider the Gaussian MIMO broadcast channel with common and private messages and obtain an outer bound for its capacity region. The crucial step in obtaining this outer

\footnotetext{
${ }^{4}$ The DoF for the private message sum rate is given by $\lim _{P \rightarrow \infty} 2\left(R_{1}+\right.$ $\left.R_{2}\right) / \log P$.
} 
bound is to construct a parallel Gaussian broadcast channel from the original channel by using the GSVD. The capacity region of the constructed parallel channel provides us the desired outer bound for the capacity region of the Gaussian MIMO broadcast channel. Next, we show that this outer bound is within a finite gap of the capacity region by comparing it with an achievable scheme, which can be obtained either by using the DPC region or by using a variation of the ZF scheme.

\section{APPENDIX}

\section{PROOF OF THEOREM 4}

To prove Theorem 4, it is sufficient to show that for any $\left(R_{0}, R_{1}, R_{2}\right) \in \mathcal{R}^{\text {out }}(P)$, we have $\left(R_{0}-\alpha_{0}, R_{1}-\alpha_{1}, R_{2}-\right.$ $\left.\alpha_{2}\right) \in \mathcal{R}^{i n}(P)$. Showing this is equivalent to proving that the following region

$$
\begin{aligned}
R_{0}^{\prime}+R_{1}^{\prime} \leq & \sum_{\ell \in \mathcal{S}_{c 2}} C\left(\frac{\tilde{h}_{1 \ell}^{2} \gamma_{\ell} P_{\ell}}{1+\tilde{h}_{1 \ell}^{2} \bar{\gamma}_{\ell} P_{\ell}}\right)+\sum_{\ell \in \mathcal{S}_{1} \cup \mathcal{S}_{c 1}} C\left(\tilde{h}_{1 \ell}^{2} P_{\ell}\right) \\
& -\alpha_{0}-\alpha_{1} \\
R_{0}^{\prime}+R_{2}^{\prime} \leq & \sum_{\ell \in \mathcal{S}_{c 1}} C\left(\frac{\tilde{h}_{2 \ell}^{2} \gamma_{\ell} P_{\ell}}{1+\tilde{h}_{2 \ell}^{2} \bar{\gamma}_{\ell} P_{\ell}}\right)+\sum_{\ell \in \mathcal{S}_{2} \cup \mathcal{S}_{c 2}} C\left(\tilde{h}_{2 \ell}^{2} P_{\ell}\right) \\
& -\alpha_{0}-\alpha_{2}
\end{aligned}
$$

$$
\begin{aligned}
\sum_{j=0}^{2} R_{j}^{\prime} \leq & \sum_{\ell \in \mathcal{S}_{c 2}} C\left(\frac{\tilde{h}_{1 \ell}^{2} \gamma_{\ell} P_{\ell}}{1+\tilde{h}_{1 \ell}^{2} \bar{\gamma}_{\ell} P_{\ell}}\right)+\sum_{\ell \in \mathcal{S}_{2} \cup \mathcal{S}_{c 2}} C\left(\tilde{h}_{2 \ell}^{2} \bar{\gamma}_{\ell} P_{\ell}\right) \\
& +\sum_{\ell \in \mathcal{S}_{1} \cup \mathcal{S}_{c 1}} C\left(\tilde{h}_{1 \ell}^{2} P_{\ell}\right)-\sum_{j=0}^{2} \alpha_{j} \\
\sum_{j=0}^{2} R_{j}^{\prime} \leq & \sum_{\ell \in \mathcal{S}_{c 1}} C\left(\frac{\tilde{h}_{2 \ell}^{2} \gamma_{\ell} P_{\ell}}{1+\tilde{h}_{2 \ell}^{2} \bar{\gamma}_{\ell} P_{\ell}}\right)+\sum_{\ell \in \mathcal{S}_{1} \cup \mathcal{S}_{c 1}} C\left(\tilde{h}_{1 \ell}^{2} \bar{\gamma}_{\ell} P_{\ell}\right) \\
& +\sum_{\ell \in \mathcal{S}_{2} \cup \mathcal{S}_{c 2}} C\left(\tilde{h}_{2 \ell}^{2} P_{\ell}\right)-\sum_{j=0}^{2} \alpha_{j}
\end{aligned}
$$

is contained in $\mathcal{R}^{i n}(P)$, where $\sum_{\ell=1}^{\left|\mathcal{S}_{1}\right|+\left|\mathcal{S}_{c}\right|+\left|\mathcal{S}_{2}\right|} P_{\ell}=\lambda_{\max }^{2} P$. By using the following fact

$C\left(\frac{x \gamma_{\ell} P_{\ell}}{1+x \bar{\gamma}_{\ell} P_{\ell}}\right)-\log \frac{\lambda_{\max }}{\lambda_{\min }} \leq C\left(\frac{x \gamma_{\ell}\left(\lambda_{\min } / \lambda_{\max }\right)^{2} P_{\ell}}{1+x \bar{\gamma}_{\ell}\left(\lambda_{\min } / \lambda_{\max }\right)^{2} P_{\ell}}\right)$

for all $x \geq 0$, and the bounds on $\alpha_{0}, \alpha_{1}, \alpha_{2}$ given by (35)-(37), one can show that the region in (88)-(91) in contained in $\mathcal{R}^{i n}(P)$, completing the proof.

\section{REFERENCES}

[1] E. Ekrem and S. Ulukus, "Degrees of freedom region of the Gaussian MIMO broadcast channel with common and private messages," in Proc. IEEE Global Commun. Conf., Dec. 2010, pp. 1-5.

[2] H. Weingarten, Y. Steinberg, and S. Shamai (Shitz), "The capacity region of the Gaussian multiple-input multiple-output broadcas channel," IEEE Trans. Inf. Theory, vol. 52, no. 9, pp. 3936-3964, Sep. 2006.

[3] H. Weingarten, Y. Steinberg, and S. Shamai (Shitz), "On the capacity region of the multi-antenna broadcast channel with common messages," in Proc. IEEE Int. Symp. Inf. Theory, Jul. 2006, pp. 2195-2199.

[4] H. Weingarten, "Multiple-input multiple-output broadcast systems," Ph.D. dissertation, Technion, Haifa, Israel, 2007.
[5] N. Jindal and A. Goldsmith, "Optimal power allocation for parallel broadcast channels with independent and common information," in Proc. IEEE Int. Symp. Inf. Theory, Jun. 2004, p. 215.

[6] A. El Gamal, "Capacity of the product and sum of two unmatched broadcast channels," Problems Inf. Transmiss., vol. 16, no. 1, pp. 3-23, Jan. 1980.

[7] R. Liu, T. Liu, H. V. Poor, and S. Shamai, "MIMO Gaussian broadcast channels with common messages," Inf. Theory Appl. Workshop Jan. 2010 [Online]. Available: http://ita.ucsd.edu/workshop/10/files/paper/ paper_1139.pdf

[8] E. Ekrem and S. Ulukus, "On Gaussian MIMO broadcast channels with common and private messages," in Proc. IEEE Int. Symp. Inf. Theory, Jun. 2010, pp. 565-569.

[9] R. H. Etkin, D. N. C. Tse, and H. Wang, "Gaussian interference channel capacity to within one bit," IEEE Trans. Inf. Theory, vol. 54, no. 12 pp. 5534-5562, Dec. 2008.

[10] A. S. Avestimehr, S. N. Diggavi, and D. N. C. Tse, "Wireless network information flow: A deterministic approach," IEEE Trans. Inf. Theory, vol. 57, no. 4, pp. 1872-1905, Apr. 2011

[11] C. Paige and M. A. Saunders, "Towards a generalized singular value decomposition," SIAM. J. Numer. Anal., vol. 18, pp. 398-405, Jun. 1981.

[12] Y. Liang, G. Kramer, and H. V. Poor, "On the equivalence of two achievable regions for the broadcast channel," IEEE Trans. Inf. Theory, vol. 57, no. 1, pp. 95-100, Jan. 2011.

[13] A. Khisti and G. Wornell, "Secure transmission with multiple antennas II: The MIMOME channel," IEEE Trans. Inf. Theory, vol. 56, no. 11, pp. 5515-5532, Nov. 2010

[14] L.-U. Choi and R. D. Murch, "A transmit preprocessing technique for multiuser MIMO systems using a decomposition approach," IEEE Trans. Wireless Commun., vol. 3, no. 1, pp. 20-24, Jan. 2004.

[15] Q. H. Spencer, A. L. Swindlehurst, and M. Haardt, "Zero-forcing methods for downlink spatial multiplexing in multiuser MIMO channels," IEEE Trans. Signal Process., vol. 52, no. 2, pp. 461-471, Feb. 2004.

Ersen Ekrem (S'08) received the B.S. and M.S. degrees in electrical and electronics engineering from Bogaziçi University, Istanbul, Turkey, in 2006 and 2007 , respectively. Currently, he is working toward the Ph.D. degree in the department of electrical and computer engineering at the University of Maryland, College Park.

He received the Distinguished Dissertation Fellowship from the ECE Department at the University of Maryland, College Park, in 2012. His research interests include information theory and wireless communications.

Sennur Ulukus (S'90-M'98) is a Professor of Electrical and Computer Engineering at the University of Maryland at College Park, where she also holds a joint appointment with the Institute for Systems Research (ISR). Prior to joining UMD, she was a Senior Technical Staff Member at AT\&T Labs-Research. She received her Ph.D. degree in Electrical and Computer Engineering from Wireless Information Network Laboratory (WINLAB), Rutgers University, and B.S. and M.S. degrees in Electrical and Electronics Engineering from Bilkent University. Her research interests are in wireless communication theory and networking, network information theory for wireless communications, signal processing for wireless communications, information-theoretic physical-layer security, and energy-harvesting communications.

Dr. Ulukus received the 2003 IEEE Marconi Prize Paper Award in Wireless Communications, the 2005 NSF CAREER Award, and the 2010-2011 ISR Outstanding Systems Engineering Faculty Award. She served as an Associate Editor for the IEEE TRANSACTIONS ON INFORMATION THEORY between 2007-2010, as an Associate Editor for the IEEE TRANSACTIONS ON COMMUNICATIONS between 2003-2007, as a Guest Editor for the Journal of Communications and Networks for the special issue on energy harvesting in wireless networks, as a Guest Editor for the IEEE TRANSACTIONS ON INFORMATION THEORY for the special issue on interference networks, as a Guest Editor for the IEEE JOURNAL ON SELECTED AREAS IN COMMUNICATIONS for the special issue on multiuser detection for advanced communication systems and networks. She served as the TPC co-chair of the Communication Theory Symposium at the 2007 IEEE Global Telecommunications Conference, the Medium Access Control (MAC) Track at the 2008 IEEE Wireless Communications and Networking Conference, the Wireless Communications Symposium at the 2010 IEEE International Conference on Communications, the 2011 Communication Theory Workshop, the Physical-Layer Security Workshop at the 2011 IEEE International Conference on Communications, the Physical-Layer Security Workshop at the 2011 IEEE Global Telecommunications Conference. She was the Secretary of the IEEE Communication Theory Technical Committee (CTTC) in 2007-2009. 